This is an author produced version of a paper published in Computational Materials Science. This paper has been peer-reviewed but does not include the final publisher proof-corrections or journal pagination.

Citation for the published paper:

Olsson, Pär; Blomqvist, Jacob. (2017). Intergranular fracture of tungsten containing phosphorus impurities : A first principles investigation.

Computational Materials Science, vol. 139, p. null

URL: https://doi.org/10.1016/j.commatsci.2017.08.018

Publisher: Elsevier

This document has been downloaded from MUEP (https://muep.mah.se) / DIVA (https://mau.diva-portal.org). 
Preprint of: Intergranular fracture of tungsten containing phosphorus impurities: A first principles investigation

By: Olsson, Pär A. T.: Blomqvist, Jakob.

Published in:

Computational Materials Science (2017), Volume 139, Pages 368-378.

DOI:

10.1016/j.commatsci.2017.08.018

Published: 2017-09-01

Citation for published version:

Olsson, P. A. T., Blomqvist, J. (2017). Intergranular fracture of tungsten containing phosphorus impurities: A first principles investigation. Computational Materials Science, 139, 368-378.

Link to published paper (free download until October 20, 2017):

https://authors.elsevier.com/a/1VehN L4--Glzu 



\title{
Intergranular fracture of tungsten containing phosphorus impurities: A first principles investigation
}

\author{
Pär A. T. Olsson ${ }^{\mathrm{a}, *}$, Jakob Blomqvist ${ }^{\mathrm{a}}$ \\ ${ }^{a}$ Materials Science and Applied Mathematics, Malmö University, SE-20506 Malmö, Sweden
}

\begin{abstract}
In the present work we have studied the influence of phosphorus impurities on the grain boundary strength of tungsten by means of quantum mechanical calculations based on density functional theory. As model grain boundary we consider the $\Sigma 5(310)[001]$ high angle configuration. The results show that by the introduction of a clean (i.e. impurity free) grain boundary in the bulk, the strength and peak stress of the cohesive zone are reduced and they are further reduced by the introduction of impurities. This effect can be attributed to the formation of polar bonds between $\mathrm{W}$ and $\mathrm{P}$, which leads to a weakening of the interface. Based on a thermodynamic analysis of the cohesive zone during the straining we find that diffusion of impurities may occur to retain thermodynamic equilibrium for constant chemical potential. This contributes to the gradual reduction of the peak stress related to fracture, which can contribute to diffusion driven delayed cracking, even when subjected to static loads.
\end{abstract}

Keywords: Tungsten, Grain boundary, Phosphorus, Density functional theory

\section{Introduction}

Owing to its high melting point, low coefficient of thermal expansion, high thermal conductivity and sputtering resistance, tungsten (W) and its alloys are considered to be the leading solid material candidates for plasma-facing components in future fusion devices such as the Tokamak reactors in the international thermonuclear experimental reactor (ITER) as well as the planned future demonstration fusion reactor (DEMO). Much of these attractive features emanate from the strong bonding between $5 d$ electrons, which results in remarkably high cohesive energy ( $8.9 \mathrm{eV} /$ atom [1]). However, a fundamental concern of using $\mathrm{W}$ for such applications is the high ductile to brittle transition temperature (DBTT) and its dependence on the microstructure and impurity concentrations [2-6]. Experiments have revealed that the DBTT for single-crystalline tungsten can be as low as $-196^{\circ} \mathrm{C}$ [7], whereas poly-crystalline samples can remain brittle up to about $800^{\circ} \mathrm{C}$ [8]. For the application of fusion reactor components, this is a major concern in light of the fact that the temperature at the armour material of the first wall and diverter under operating conditions typically lie in the range $\sim 600-900^{\circ} \mathrm{C}$, which in principle means that the first wall armour material is at risk of rupturing because of brittle fracture $[2,9,10]$. This is of particular importance for the thin layer tungsten diverter surface, which is subjected to significant thermal gradients, resulting in stresses that can reach hundreds of MPa at regular operation and even higher stresses when subjected to transient loads such as plasma disruptions $[9,10]$.

To explain the wide range of DBTT, a number of experimental studies have been performed to bring insight on the mech-

\footnotetext{
${ }^{*}$ Corresponding author

Email address: Par.0lsson@mah.se (Pär A. T. Olsson)
}

anisms responsible for its behaviour. It has been deduced that the DBTT is strain rate dependent and there is a strong correlation with the activation energy associated with screw dislocation mobility. Moreover, the introduction of impurities increases the activation energy, implying further reduced dislocation mobility for impure tungsten which is attributed to impurity segregation at the dislocation core inhibiting the movement $[11,12]$. The DBTT is expected to be further increased while in operation, because of the cascade damage following the unprecedented degree of radiation that the material will be subjected to during operation. Moreover, transmutation of tungsten into rhenium or osmium may contribute to precipitation hardening [13]. These events collectively will result in a change in the microstructure with increased vacancy, self-interstitial and dislocation densities along with formed precipitates that further reduce the ductility and inhibits dislocation movement [2].

Because of the high melting temperature $\left(\sim 3400^{\circ} \mathrm{C}[1]\right)$, tungsten products are commonly fabricated through the employment of powder technology, rather than melting or casting. Thus, most tungsten materials for practical applications have a poly-crystalline microstructure. To gain insight on the fracture behaviour of poly-crystalline tungsten, experimental studies have been performed to compare the fracture behaviour of single- and technically pure poly-crystals [12, 14]. These works have shown that cleavage failure along the grain boundaries limits the fracture toughness in poly-crystalline $\mathrm{W}$ and the explanation commonly cited is that segregation of impurities at grain boundaries reduces the grain boundary strength and results in mode I type cleavage as opposed to ductile failure. In particular, it has been believed the main cause of this behaviour can be attributed to impurities of sulphur $(\mathrm{S})$, oxygen $(\mathrm{O})$ and phosphorus $(\mathrm{P})$ segregated at the grain boundaries, whereas car- 
bon (C) and boron (B) impurities improves the strength [2]. Of these elements, especially $\mathrm{P}$ has been deemed to be the main culprit of the grain boundary embrittlement of technically pure tungsten. This conjecture has been motivated by an observed correlation between loss in ductility and increasing $\mathrm{P}$ content [15]. However, recent experimental works have indicated that impurity free grain boundaries also may preferentially rupture by cleavage [16]. Thus, there are contradicting experimental observations, which could imply that even impurity-free grain boundaries may undergo brittle failure. Therefore the mechanisms responsible for promoting grain boundary cleavage are not fully understood and the degree to which different element impurities weaken the grain boundaries remains elusive. Also the question of whether all the aforementioned impurities affect the grain boundary strength is unanswered, which merits further investigation.

To gain insight on the effect of impurities on the physical and mechanical properties of materials, $a b$ initio methods such as quantum mechanical density functional theory (DFT) serve as useful tools to predict the behaviour. Such modelling can be used to predict ideal fracture mechanical properties related to transgranular and intergranular fracture, cf. for instance [1721] and the references therein. For modelling grain boundary cohesion of body-centered-cubic (BCC) metals, owing to its industrial importance, iron grain boundaries have been the scope of numerous ab initio modelling efforts [22-28] to understand the influence of impurities and alloying elements on the grain boundary properties. Although not studied to the extent as to iron, because their electronic structures differs substantially from iron, impurity inhabited grain boundaries in molybdenum [29-31] and niobium [30, 31] have been modelled by means of DFT to elucidate how the electronic structure affects the grain boundary cohesion.

With respect to tungsten, semi-empirical works addressing impurity embrittlement were performed by Krasko and coworkers [32, 33], who studied the influence of impurities $(\mathrm{N}$, $\mathrm{O}, \mathrm{P}, \mathrm{S}, \mathrm{Si}, \mathrm{B}$, and $\mathrm{C}$ ) on the cohesion of $\Sigma 3(111)$ type grain boundaries. It was reported that, with the exception $\mathrm{B}$ and $\mathrm{C}$, all considered impurities contribute to a weakening of the grain boundary. Boron and carbon, however, were found to improve the grain boundary cohesion. In light of the potential application of tungsten as plasma-facing material, recently some studies have been conducted to investigate the embrittling potency of impurities by means of DFT modelling in conjunction with the ideal grain boundary fracture energy criterion formulated by Rice and Wang [34]. For these applications $\mathrm{H}$ and He isotopes are of particular interest, which based on the Rice-Wang criterion have been found to reduce the grain boundary fracture energy [35, 36]. Moreover, it has been predicted that $\mathrm{H}$ impurities lower the peak stress required for decohesion [35]. Similar modelling has been employed to identify potential transition metal candidates to improve the grain boundary strength [37-39]. Such works have revealed that solutes located at the centre of the $d$-band in the periodic system are beneficial for improving the grain boundary strength [39]. Regarding P impurities in W, based on the Rice-Wang formalism it has been observed that the introduction of impurities leads to grain bound- ary weakening [40]. Moreover, by separating the mechanical and chemical effects, a recent DFT-based work [41] has implied that the small lattice parameter and the strong bonding of tungsten yield an enhanced embrittelling effect when impurities are introduced, compared to other BCC metals.

Despite that previous DFT-based works, in accordance with experimental results, have established that $\mathrm{P}$ impurities lead to an embrittlement of tungsten grain boundaries - detailed investigation of the gradual reduction in grain boundary strength during the separation and the cleavage behaviour remains. Thus, the purpose of the present work is to study the influence of $\mathrm{P}$ impurities on the grain boundary strength by means of DFT modelling. In particular, we aim to investigate how they affect the peak stress associated with decohesion and Griffith work of fracture, and how the impurity transport influences the cohesive strength during the mode I opening based on a thermodynamic description of the equilibrium impurity coverage. This will give qualitative insight to the influence of $\mathrm{P}$ atoms on the cohesive strength of grain boundaries and provide tractionseparation data that can be used for macroscopic cohesive zone modelling of intergranular fracture in tungsten. Moreover, as part of this work we investigate how the charge distribution is altered by the addition of $\mathrm{P}$-impurities in the grain boundary, to study the electronic mechanisms behind the impurity induced grain boundary weakening.

The paper is organised as follows: In the following section, continuum modelling of brittle fracture and decohesion are discussed. This is followed by a description of the computational methods used in this paper and a disposition of the obtained results. Finally, the paper ends with a short summary of the observations and conclusions.

\section{Cohesive zone modelling of cleavage fracture of grain boundaries}

For fracture events displaying limited plasticity the cohesive zone approach is a useful tool for modelling the interfacial energetics and traction-separation behaviour in the process zone $[42,43]$. The total response of the solid can be described in terms of the cohesive zone model representing the interfacial strength and the elastic response of the underlying atomic planes. To extract the cohesive zone parameters based on firstprinciples modelling it is necessary to assume that the inclination between the decohering planes is small such that the crack opening can be described by the separation of essentially parallel planes. For the case of brittle decohesion this is a good approximation in the local proximity of the decohering site and gives insight to the fracture properties of the material.

For modelling brittle fracture of grain boundaries in solids, it is beneficial to consider the interface as a cohesive zone embedded in an elastic medium. This encourages the employment of excess properties such as proposed by Gibbs for describing interfaces [44-47]. Through this approach the excess properties can be used to represent inhomogeneities such as interfaces and grain boundaries in the material. The excess energy of a decohering grain boundary can be defined as the difference between the total energy of the fracturing solid and that of an ideal 
bulk. This definition suggests that the surrounding crystalline regions retain bulk properties and that the fracture is localised at the interface. The excess energy is described by a potential $\phi(\delta, \theta)$ that gives the strain energy in terms of the interplanar separation, $\delta$, and the impurity coverage, $\theta$.

Owing to the fact that impurities may be attracted to regions of high stresses, it may be necessary to account for impurity diffusion towards the crack tip when computing the cohesive zone properties. Such diffusion leads to varying degrees of impurity coverage for the gradually separating crack surfaces and it can occur either via the free crack surfaces or through point defect clusters forming as a result of the irradiation damage accumulated while in service. This becomes important when the crack growth occurs much slower than the impurity motion and to account for it, the modelling needs to be performed at constant chemical potential. To ensure chemical equilibrium it is necessary that the chemical potential of impurities within the cohesive zone matches that of the impurity reservoir from which the diffusing impurity atoms emanate [46]. Through Legendre transforms the grand force potential to be minimised can be defined as

$$
\Phi(\sigma, \mu, T)=\phi-T S / A-\sigma \delta-\mu \theta / A
$$

where $S$ is the entropy, $\mu$ is the chemical potential, $\theta$ is the impurity concentration and the stress is represented by $\sigma=\partial \phi / \partial \delta$ $[46,48]$. Typically, to compute the effects of temperature it is necessary to resort to statistical mechanical approaches, such as cluster expansion techniques [49-51], to account for configurational entropic contributions. Moreover, the vibrational entropy can be accounted for through the quasi-harmonic approximation, which relies on the computed phonon density of states [52]. However, in the present work we neglect the entropic contributions to (1).

\section{Simulation setup and methodology}

\subsection{Numerical details}

All DFT simulations in this work are performed using the well-established Vienna ab initio simulation package (VASP) [53-56]. For modelling the electron-ion interaction we adopt the frozen core projector-augmented-wave (PAW) method [57, 58 ] with the electron descriptions for $\mathrm{W}$ and $\mathrm{P}$ comprising the $5 d^{4} 6 s^{2}$ and $3 s^{2} 3 p^{3}$ orbitals, respectively. Because the van der Waals (vdW) interaction is of great importance to describe the ground state of phosphorous, for assessing the exchange and correlation contribution in all simulations we adopt the nonlocal optB88-vdW exchange-correlation functional described by Dion et al. [59, 60] and implemented by Klimes et al. $[61,62]$. It is well established that vdW-based exchangecorrelation functionals or corrections are necessary to accurately capture for instance the adsorption of vdW-dominated compounds onto metal surfaces [63]. Moreover, such strategies are required for an accurate account of the crystallography of many phosphorus morphologies and their cohesive energy $[64,65]$, whereas conventional approaches fail to describe the interaction. Although we expect the vdW-interaction to be relatively small in the present application, we utilise the optB88vdW exchange-correlation functional to ensure an improved description of the phosphorus ground state properties.

In order to obtain satisfactory results we have converged the kinetic energy cutoff for the plane-wave basis set and $k$-point density such that the ground state energy is converged within $1.0 \mathrm{meV} /$ atom. To achieve this, we have used a kinetic energy cutoff of $400 \mathrm{eV}$ and a $k$-point grid for the first Brillouin zone of the primitive tungsten BCC cell corresponding to a gammacentered $16 \times 16 \times 16$ sized grid generated by means of the Monkhorst-Pack method [66]. The $k$-point mesh for the supercells used in the decohesion simulations are made commensurate with the primitive $\mathrm{BCC}$ cell. In order to prevent numerical issues with lack of convergence from occurring, we use smearing of the Brillouin zone integration based on the MethfesselPaxton scheme [67]. The adopted smearing width used in the calculations corresponds to a broadening of $0.2 \mathrm{eV}$, which was found to give satisfactory convergence and accuracy.

\subsection{Ground state calculations and supercell convergence}

Tungsten is a group VIB refractory metal with a ground state structure corresponding to a BCC crystal with lattice parameter $a_{0}=3.165 \AA$ [68], cf. Figure 1(a). To ensure that the DFT modelling accurately predicts the ground state we perform fully relaxed calculations in which both the size and shape of the primitive BCC cell are allowed to vary in order to find a stress free equilibrium state, whereby the optimised cell dimensions are evaluated and compared with the experimentally measured lattice parameter. For phosphorus there are different configurations that can be used as a reference for the ground state, including many minerals. For benchmarking the adopted modelling approach, in the present work we consider two different configurations for pure phosphorus: bulk black phosphorus (A17 lattice) and an isolated $\mathrm{P}_{4}$-molecule. Experimental observations have revealed that black phosphorus has a ground state configuration corresponding to an orthorhombic crystal with $a=3.313 \AA, b=10.473 \AA$ and $c=4.374 \AA$, with $u=0.1034$ and $v=0.0806$ [69], see Figure $1(\mathrm{~b}) . \mathrm{P}_{4}$ is a molecule containing four $\mathrm{P}$ atoms ordered in a tetrahedral manner [70], see Figure 1(c). The experimentally observed bond length for such molecules corresponds to $2.195 \AA$. To compute the ground state energy of an isolated $\mathrm{P}_{4}$-molecule, it is placed at the centre of a large supercell of size $10 \AA \times 10 \AA \times 10 \AA$, whereafter the atomic positions are optimised while the supercell size and shape constrained to remain unchanged. This way the interaction with the molecules in the neighbouring periodic cells is limited, and the computed ground state energy can be considered to be that of an isolated molecule.

The grain boundary considered in this work corresponds to a $\Sigma 5(310)$ [001] configuration, which is illustrated in Figure 1(d). This grain boundary is a symmetric tilt boundary with the rotation angle corresponding to $36.9^{\circ}$ about the [001]-rotation axis, which is commonly observed experimentally in group VB and VIB refractory metals [71-73]. The number of atomic layers to be utilised in the normal direction of the grain boundary was evaluated by studying the grain boundary energy for 20 and 


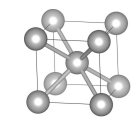

(a)

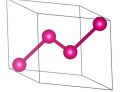

(b)

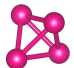

(c)

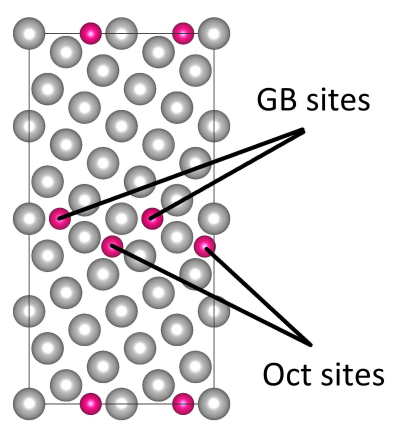

(d)

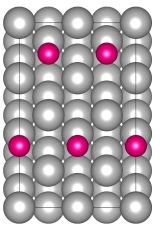

(e)
Figure 1: Illustration of the ground state structures for (a) W, (b) black P and (c) $\mathrm{P}_{4}$. (d) Schematic illustration of the $\Sigma 5(310)$ [001] interface with grain boundary and octahedral impurity sites indicated. (e) The $\Sigma 5(310)[001]$ grain boundary plane indicating possible impurity sites.

24 (310)-layers, i.e. 80 and $96 \mathrm{~W}$-atoms, respectively. Because interface induced stresses may affect the grain boundary planar dimensions, for the grain boundary relaxation, the supercell is prevented from relaxing fully to avoid the grain boundary in-plane dimensions to differ from that of a perfect bulk. By adopting this approach we reduce the impact of finite size effects and ensure that any deformations are purely one-dimensional. Thus, we only allow the supercell to relax in the grain boundary normal direction. To this end, a variety of simulations are performed for different cell dimensions in the [310]-direction, whilst allowing all atomic coordinates to relax to find the minimum. Moreover, to account for the fact that the lowest grain boundary configuration may have broken mirror symmetry [74], we impose relative shearing of the upper and lower grains to obtain the lowest grain boundary energy configuration.

\subsection{Impurity solution}

To investigate the preferential solution sites for $\mathrm{P}$ in $\mathrm{W}$ we study four different potential cases: (i) $\mathrm{P}$ situated at an octahedral interstitial site, (ii) $\mathrm{P}$ situated at a tetrahedral interstitial site and (iii) $\mathrm{P}$ as a substitutional atom. Finally we also consider (iv) $\mathrm{P}$ situated in the grain boundaries such as depicted in Figures 1(d)-(e).

Cases (i)-(iii) correspond to modelling the solution of $\mathrm{P}$ in bulk W. In order to reduce the interaction with mirror impurities in neighbouring periodic cells it is necessary that the supercell has sufficient size. The interstitial energy of a tetrahedral $\mathrm{P}$ interstitial was converged with respect to the supercell size, which revealed that a supercell corresponding to $4 a_{0} \times 4 a_{0} \times 4 a_{0}$ was of sufficient size to quantitatively determine the preferred impurity sites. For the simulations of impurity defect formation energy, all atoms are allowed to relax, such that they may relocate to equilibrium sites. Moreover, to account for the fact that an atom of a different element has entered the supercell, both size and shape of the supercell are allowed to vary in order to minimise the stress. Following Scheiber et al. [40], we compute the difference between formation energy of substitutionals and interstitials using the relation

$$
\Delta E^{b}=E_{\text {sub }}-\left(E_{\text {int }}-E_{\text {host }}\right)
$$

where $E_{\text {sub }}$ and $E_{\text {int }}$ are the supercell energies of systems containing a substitutional and interstitial, respectively and $E_{\text {host }}$ is the energy of the perfect BCC primitive cell. Because we consider two different interstitials we need to compute $\Delta E^{b}$ for both the tetrahedral and octahedral types. If $\Delta E^{b}$ is negative, the impurity is preferentially situated as a substitutional, whereas a positive value implies an interstitial preference.

To compute the segregation energy in the bulk we adopt the definition from [40], in which it is defined as

$$
E_{S}^{b}=E_{\text {sub }}-\left((N-1) E_{\text {host }}+E_{P}\right)
$$

for a substitutional and for an interstitial

$$
E_{S}^{b}=E_{\text {int }}-\left(N E_{\text {host }}+E_{P}\right)
$$

where $N$ is the number of $\mathrm{W}$ atoms in the perfect supercell and $E_{P}$ is the ground state energy per atom for the black phosphorus structure.

When studying the influence of P-impurities situated at the grain boundaries, they are placed in the voids that form in the grain boundaries and at octahedral sites in its proximity as illustrated in Figure 1(d) and (e), whereafter relaxation ensues for the impurity atoms to relocate to equilibrium sites. To compute the grain boundary segregation energy, $E_{S}^{G b}$, we use the relation

$$
E_{S}^{G b}(\theta)=E^{G b}(\theta)-E^{G b}(\theta-1)-\min \left(E_{S}^{b}\right)-E_{P}
$$

in which $E^{G b}(\theta)$ is the ground state energy of a supercell containing $\theta$ P-impurities per grain boundary and $\min \left(E_{S}^{b}\right)$ represents the minimum energy of (3) and (4).

Since the main scope of this work is to qualitatively study the influence of impurities on the mechanical properties of grain boundaries, seven degrees of gradually increasing impurity coverage are considered: one without any impurities $(\theta=0)$ and gradually increasing the coverage to contain up to six impurity atoms $(\theta=1-6)$ to account for the fact that upon cleavage the free surfaces may be subject to increased coverage following impurity transport. The first four impurity atoms are placed sequentially in the voids of the grain boundary, see Figure 1(e), and the additional two are placed at octahedral sites immediately next to the grain boundary, see Figure 1(d). A more complete description would be to model multiple intermediate configurations by considering larger supercells for instance through coarse grained techniques such as cluster expansion modelling [49-51]. Because we in the present work view the crack opening as the gradual separation between layers accompanied by impurity diffusion to cover the free surfaces, the considered impurity configurations are sufficient to give a qualitative description of the cohesive zone properties for the system at hand.

\subsection{Decohesion}

For the decohesion, the property of particular interest is the excess energy curve, which can be used to evaluate cohesive 
zone properties associated with grain boundaries, including the peak stress and the Griffith work of fracture. To extract such properties we adopt the strategy outlined by Van der Ven and co-workers $[44,46]$. In this approach the grain boundary is seen as an anomaly from the otherwise homogeneous crystalline material. Thus, in order extract the deviating behaviour triggered by the grain boundary, the response of the defect free crystal needs to be mapped out. For this purpose we perform relaxed $a b$ initio tensile simulations of a perfect crystal strained in the [310]-direction. In the literature $[44,46]$ it is well documented that such simulations have more than one potential equilibrium state, depending on whether the initial state supercell is subjected to an evenly distributed strain or if the strain is localised between two atomic planes. In the present work both possibilities are accounted for by probing the energy vs. displacement for both tensile strategies and then for each strain increment it is assumed that the prevailing ground state configuration is that which exhibits the lowest energy.

To extract the excess energy of the defect free bulk crystal, it is assumed that the interplanar interaction can be described by an interplanar potential $\phi^{b}$ [75]. Hence, if the supercell contains $n$ [310]-layers and is subjected to an elongation, $\delta_{g}$, the total strain energy, $\phi^{g}$, can be described by

$$
\phi^{g}\left(\delta_{g}\right)=(n-1) \phi^{b}\left(\delta_{1}\right)+\phi^{b}\left(\delta_{2}\right)
$$

where $\delta_{2}$ and $\delta_{1}$ represent the interplanar stretch of the excess region and the surrounding bulk, respectively. This means that $\delta_{2}$ accounts for stretch associated with the interface and that the remaining atomic planes are assumed to be uniformly separated by $\delta_{1}$. Strictly this is an idealisation because the stretch of the atomic layers in the proximity of the interface will not necessary correspond to $\delta_{1}$. However, the energy contributions associated with this minor deviation is incorporated as a part of the excess potential. Below the inflection point, where the strain energy of the evenly strained configuration is less or equal to that of a cleaved configuration, $\delta_{1}=\delta_{2}$ and $\delta_{g}=n \delta_{1}$. However, when the supercell elongation exceeds beyond the inflection point, cleavage has started to occur, which implies $\delta_{1}<\delta_{2}$. In that case the relation between $\delta_{1}$ and $\delta_{2}$ can be obtained by the fact that there is equilibrium between all atomic planes and the interplanar stress corresponds to that in the supercell, i.e. $\partial \phi^{b} / \partial \delta_{1}=\partial \phi^{b} / \partial \delta_{2}=\partial \phi^{g} / \partial \delta_{g}$.

The excess energy is assumed to have the functional format of an extended version of Rose's universal binding energy relation (xUBER) $[46,76,77]$, i.e.,

$$
\phi(\delta)=\phi_{0}+C \delta_{c}^{2}\left[1-\left(\sum_{m=0}^{m_{\max }} \alpha_{m}\left(\frac{\delta-\delta_{0}}{\delta_{c}}\right)^{m}\right) \exp \left(-\frac{\delta-\delta_{0}}{\delta_{c}}\right)\right]
$$

which is truncated by assuming that $m_{\max }=6$. Moreover, to ensure consistency with the original UBER format [76, 77] we assign the parameters $\alpha_{0}=\alpha_{1}=1$ and $\alpha_{2}=0$, while no restrictions are made for the remaining parameters, which are allowed assume arbitrary values to improve the fit. The numerical fitting is performed by using the Nelder-Mead downhill simplex algorithm [78] initiated at different starting points to ensure that an optimal solution is found.
To compute the excess for supercells containing a grain boundary, and possibly impurities, it is necessary to fix some atom layers such that not the entire supercell is subjected to strain. The reason for this is that the studied system should only contain a single grain boundary that undergoes deformation. To overcome this obstacle we simply constrain five layers surrounding one of the grain boundaries to be fixed, which ensures that only one of the grain boundaries is subjected to strain and contributes to the strain energy.

To extract the excess energy related to the grain boundary we use a similar strategy as for the bulk. If the cleavage occurs at the grain boundary, the strain energy can be expressed as

$$
\phi^{g}\left(\delta_{g}\right)=n \phi^{b}\left(\delta_{1}\right)+\phi^{G b}\left(\delta_{2}\right)
$$

where $\phi^{G b}$ is the excess energy for a grain boundary. By using the knowledge of the bulk excess, the grain boundary counterpart can be extracted by using that the stress in the supercell corresponds to that between all atomic layers. Thus, we get $\partial \phi^{g} / \partial \delta_{g}=\partial \phi^{G b} / \partial \delta_{2}=\partial \phi^{b} / \partial \delta_{1}$ and $n \delta_{1}+\delta_{2}=\delta_{g}$. Due to the prior knowledge of $\phi^{b}$, this energy contribution can be removed from the total energy, $\phi^{g}$, which enables the extraction of the grain boundary excess, $\phi^{G b}$, as implied by Eq. (8).

\section{Results and discussion}

\subsection{Ground state properties of $B C C W$, black $P, P_{4}$ and $\Sigma 5(310)$ [001] grain boundary}

To benchmark the predictability of the DFT modelling, through geometrical optimisations of the size and shape of the BCC unit cell, we have evaluated the lattice parameter for pure tungsten and compared with experimental data. The optimised lattice parameters for $\mathrm{W}$ is equal to $3.180 \AA$ which deviates by less than one percent from the experimentally measured value of $3.165 \AA$ [68]. Good agreement with experimental data for the considered P-phases is highly dependent on the assumed exchange-correlation functional. Previous works [65] have indicated that conventional GGA-PBE calculations $[79,80]$ can predict lattice parameters that deviate by about $10 \%$ from experimental data. However, by adopting the optB88-vdW exchange-correlation functional our results are substantially improved. The relaxed lattice parameters for black $\mathrm{P}$ correspond to $a=3.314 \AA, b=10.478 \AA$ and $c=4.376 \AA$, with $u=0.1036$ and $v=0.0785$, which agree very well with experimental data [69]. Likewise, the P-P bond length for tetrahedral $\mathrm{P}_{4}$ predicted by DFT modelling is found to be $2.214 \AA$, which concurs well with the bond length $2.195 \AA$ as observed in gas-phase electron diffraction measurements [70]. Thus, typical deviations are less than one percent, which indicate good predictive characteristics of the assumed DFT modelling. The computed ground state energy difference between black phosphorus and $\mathrm{P}_{4}$ was found to be $0.30 \mathrm{eV} /$ atom in favour of the black phosphorus phase.

To ensure well-converged results we studied how the supercell size affect the results. This convergence study revealed that the grain boundary energies for 20 and 24 layers only differed by less than $0.02 \mathrm{~J} / \mathrm{m}^{2}$, which are acceptable results for the 

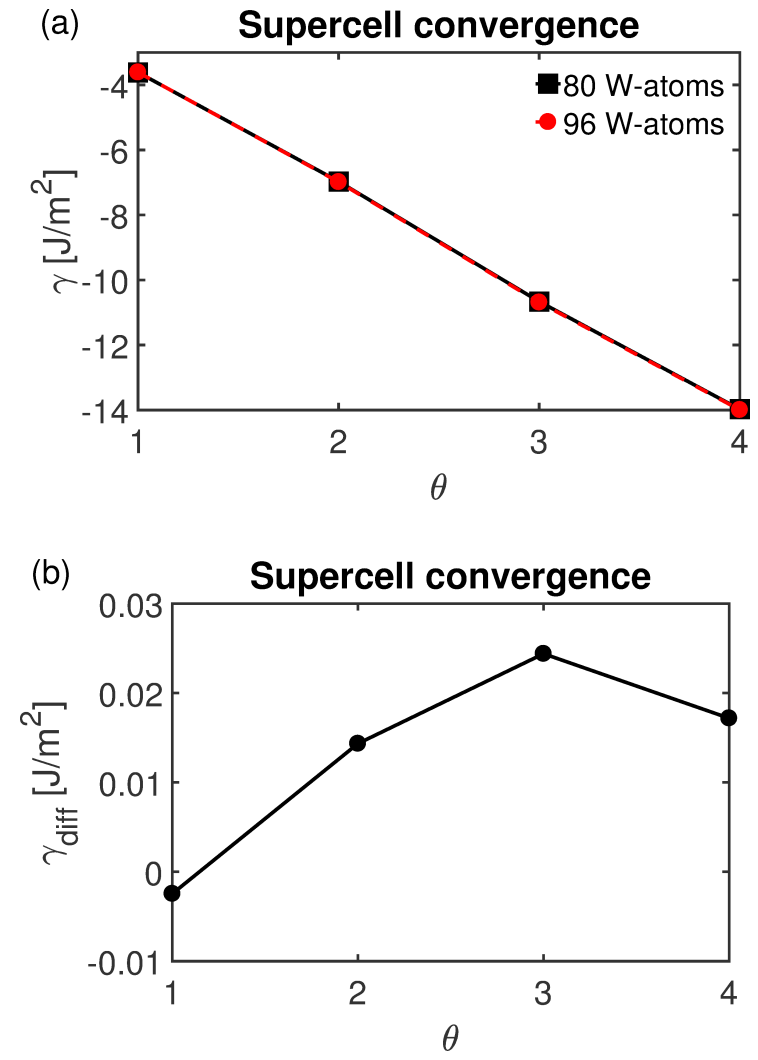

Figure 2: (a) Supercell ground state energy variation for supercell sizes containing 80 and 96 atoms, with the reference energy corresponding to the impurity free grain boundary. (b) The difference in ground state energy between the supercells containing 80 and 96 atoms for different impurity coverages, which corresponds to the difference between the two curves in (a).

present study. Moreover, we gradually introduced P-impurities in the mirror symmetric grain boundary and studied how the ground state energy varies with concentration, see Figure 2(a) and (b). From Figure 2(a) it is seen that the change in energy are very similar for the 20 and 24 layer systems. By taking the difference between the two curves, see Figure 2(b), it is seen that they differ by less than $0.03 \mathrm{~J} / \mathrm{m}^{2}$. This leads to the conclusion that the choice of the supercell for the grain boundary calculations to consists of 20 (310)-layers and a total of 80 tungsten atoms is of sufficient size for the present investigation. Thus, the ideal (i.e. unrelaxed) dimensions of the supercell correspond to $2 a_{0}$ in the [001]-direction, $2 \sqrt{10} a_{0}$ in the [310]-direction and $\sqrt{10} a_{0}$ in the [130]-direction.

The $\Sigma 5(310)$ [001] grain boundary is a relatively open grain boundary type, which means that it may act as a trapping site for impurities and it has a higher grain boundary energy than more closed types. It is of interest to investigate how the supercell relaxes following the introduction of the grain boundary to get an indication whether the grain boundary opens or closes up because of the relaxation. In accordance with previous DFT works on tungsten $\Sigma 5$-grain boundaries [39, 40, 74] the lowest energy configuration corresponds to a state where the neighbouring grains are shifted in the [001]-direction such that the mirror symmetry is broken. Specifically, the shift is found to be $0.18 a_{0}$ in the [001]-direction, while the supercell increases in size because of the relaxation by $0.27 \AA$ in the normal direction of the grain boundary. The computed grain boundary energy for $\theta=0$ corresponds to $2.5 \mathrm{~J} / \mathrm{m}^{2}$, which concurs with the findings in $[40,74]$.

\subsection{Impurity solution}

Experiments have indicated that phosphorus is in general virtually insoluble in tungsten, which is why tungsten is known to only contain of the order of $10 \mathrm{wppm}$ of the impurity [16]. To ensure that the adopted DFT modelling predicts these characteristics, we have computed the solution energy for impurities in the bulk as octahedral and tetrahedral interstitials. Using (2), we get that $\Delta E^{b}$ is negative for both interstitial types. Specifically they become -4.3 and $-6.3 \mathrm{eV} /$ atom for the octahedral and tetrahedral interstitials, respectively. Because of the negative character of these energies, it is concluded that it is highly unlikely that $\mathrm{P}$ is preferentially situated in bulk tungsten as interstitials, instead they will be situated as substitutionals. The segregation energy for a P-impurity to occupy an existing vacancy in the bulk corresponds to $1.01 \mathrm{eV} /$ atom.

A similar investigation was performed to study the impurity segregation in the grain boundary. It was found that the grain boundary segregation energy for a grain boundary with $\theta=1$, calculated by means of Eq. (5), is equal to $-2.25 \mathrm{eV} /$ atom. For the increased impurity content of $\theta=2, \theta=3$ and $\theta=4$ it is found to be $-2.69,-2.17$ and $-2.15 \mathrm{eV} /$ atom, respectively. For $\theta=5$, we introduce an impurity atom at an octahedral site right below the grain boundary, see Figure 1(d). The segregation energy for such an impurity corresponds to $0.30 \mathrm{eV} /$ atom. This implies that the population of $\mathrm{P}$ in the equilibrium grain boundary up to $\theta=4$ leads to a lowering of the grain boundary energy, which means that it is a plausible trapping site for phosphorus. The positive character for the octahedral sites in the proximity of the grain boundary, implies that they are not likely trapping sites for unstressed systems. These findings concur with experimental observations and explains why the fraction of $\mathrm{P}$ in tungsten generally is found only in low concentrations.

In light of the fact that the most likely occurrence of $\mathrm{P}$ is in grain boundaries, we consider this type of trap site to represent the reservoir of impurity atoms that will migrate to the cohesive zone as it is stretched. Thus, as an estimate for the chemical potential, we use the relation

$$
\mu=\frac{1}{4} \sum_{\theta=1}^{4}\left[E^{G b}(\theta)-E^{G b}(\theta-1)\right]
$$

to define the chemical potential of $\mathrm{P}$ in $\mathrm{W}$.

\subsection{Binding electron density and Bader charge analyses}

To gain insight on the grain boundary cohesion character we study the bonding electron density for $\theta=4$ and the charge transfer between $\mathrm{W}$ and $\mathrm{P}$. In the present work the bonding electron density is defined as the difference in charge distribution between the impurity inhabited grain boundary and that of the impurity free grain boundary and impurity sublattices modelled as separate supercells. Figures 3(a) and (b) reveal that there is 

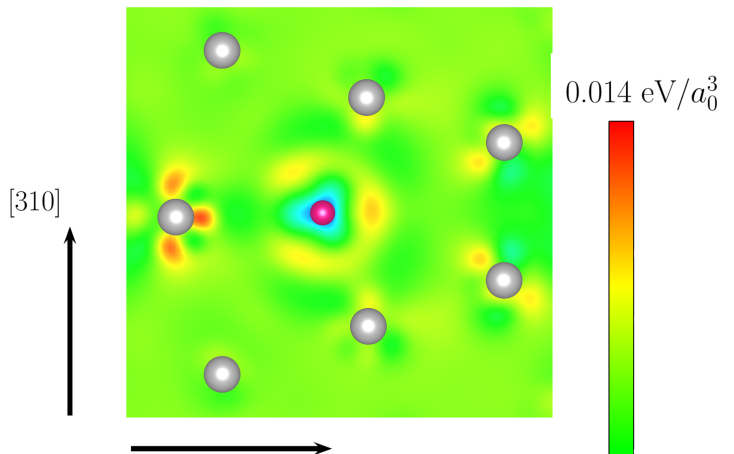

(a)

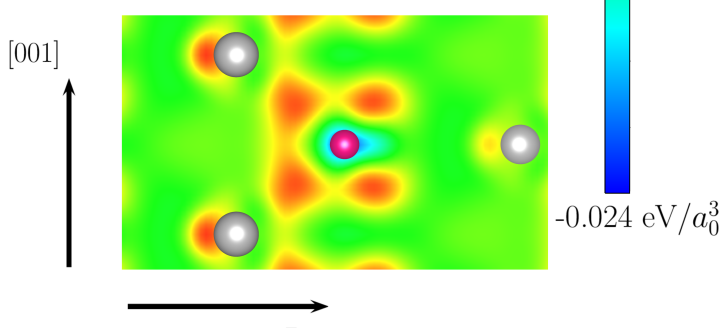

$\lceil 1 \overline{3} 0]$

(b)

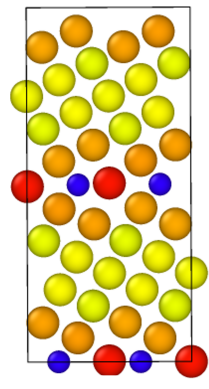

(c)
0.35

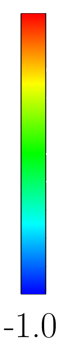

$-1.0$

Figure 3: Contour plots of the bonding electron density for the $\theta=4$ system projected onto the (a) [001]- and (b) [310]-planes in the grain boundary. Bader charge analysis for (c) $\theta=4$. The atom color represents the change in charge following the introduction of phosphorus in the grain boundary - a negative Bader charge transfer implies that the ion becomes negatively charged.

a negative charge accumulation in the close proximity of the impurity atom and a reduction of electron density in its immediate surroundings. This is in line with the fact that phosphorus has a higher electronegativity than tungsten [81]. To investigate this closer we performed a Bader charge analysis [82] using the open source BADER code [83]. The analysis reveals that there is a charge transfer from the surrounding tungsten to the phosphorus in the grain boundary, see Figure 3(c). Specifically, it is found that the change in charge for the $\mathrm{P}$ atoms correspond to -1.0 , which implies that phosphorus act as negatively screened ions in the grain boundary.

Cottrell [84] proposed a unified theory that implies that impurities that form covalent bonds with the matrix improves intergranular cohesion, whereas negatively screened ions, which do not form covalent bonds, weakens the cohesion. The Bader

and charge density analyses reveal that the charge density between atoms is not increased, but rather reduced in the proximity of the impurity atom, which implies that no significant covalent bonds are formed between the impurity and the matrix. Thus, the bonding is of polar character and our predictions of $\mathrm{P}$ contributing to the grain boundary embrittlement in $\mathrm{W}$ is in line with those of Cottrell.

\subsection{Decohesion}

To determine the excess for the bulk system we conducted tensile simulations in the [310]-direction, see Figure 4(a). The modelling shows that, initially the strain energy follows a linear elastic behaviour in accordance with Hooke's law. However, as the strain increases the separation becomes too large for the material to heal and two surfaces are created, as indicated by the vertical dashed lines in Figure 4. The total obtained surface energy for the created surfaces corresponds to the Griffith work of fracture, which appears in the asymptotic limit of large strains and amounts to about $7.6 \mathrm{~J} / \mathrm{m}^{2}$ for the present orientation. We note that the supercell strain energy depends on the number of free layers in the model $[47,75]$, while the excess strain energy is invariant to the number of layers available in the model. To extract the bulk excess, $\phi^{b}$, we fitted the supercell strain energy (Eq. (6)) to the curve in Figure 4(a) by using the xUBER. As seen in Figure 4(a), the fitted curve corresponds well with the DFT data, which demonstrates that the adopted xUBER is a suitable parametrisation of the excess energy. The fitted parameters for $\phi^{b}$ can be seen in Table 1 .

Before investigating the effect of impurities on the grain boundary cohesion, we have studied the tensile behaviour of impurity free $(\theta=0)$ grain boundaries. The observations are similar to those found for the perfect bulk, i.e. for small strains the behaviour follows Hooke's law, while as the strain increases the convexity of the strain energy curve is lost and the material ruptures, see Figure 4(b). It should be noted that the minimum energy does not appear at zero strain when grain boundaries are considered. The reason for this seemingly odd choice of reference is motivated by the fact that we need to measure the strain from the perfect unrelaxed configuration, meaning that the reference state does not correspond to a stress free state. This choice of reference is necessary in order to compare the grand force potential for the grain boundary with varying degrees of impurity coverage and to accurately predict at which stress the equilibrium coverage increases. The zero stress state corresponds to an elongation of the supercell in the normal direction of the grain boundary of $0.27 \AA$, which was the previously observed elongation of the minimum energy state. The strain energy curve in Figure 4(b) reveals that the introduction of a grain boundary reduces the fracture energy from $7.6 \mathrm{~J} / \mathrm{m}^{2}$ to about $5.9 \mathrm{~J} / \mathrm{m}^{2}$, suggesting a slight lowering in the fracture strength of the material. The introduction of impurities yields a lowering in the work of fracture, which can be seen in figures 4(c)-(h), where the asymptotic large separation energies decrease with increasing impurity content. For $\theta=1-4$ the 

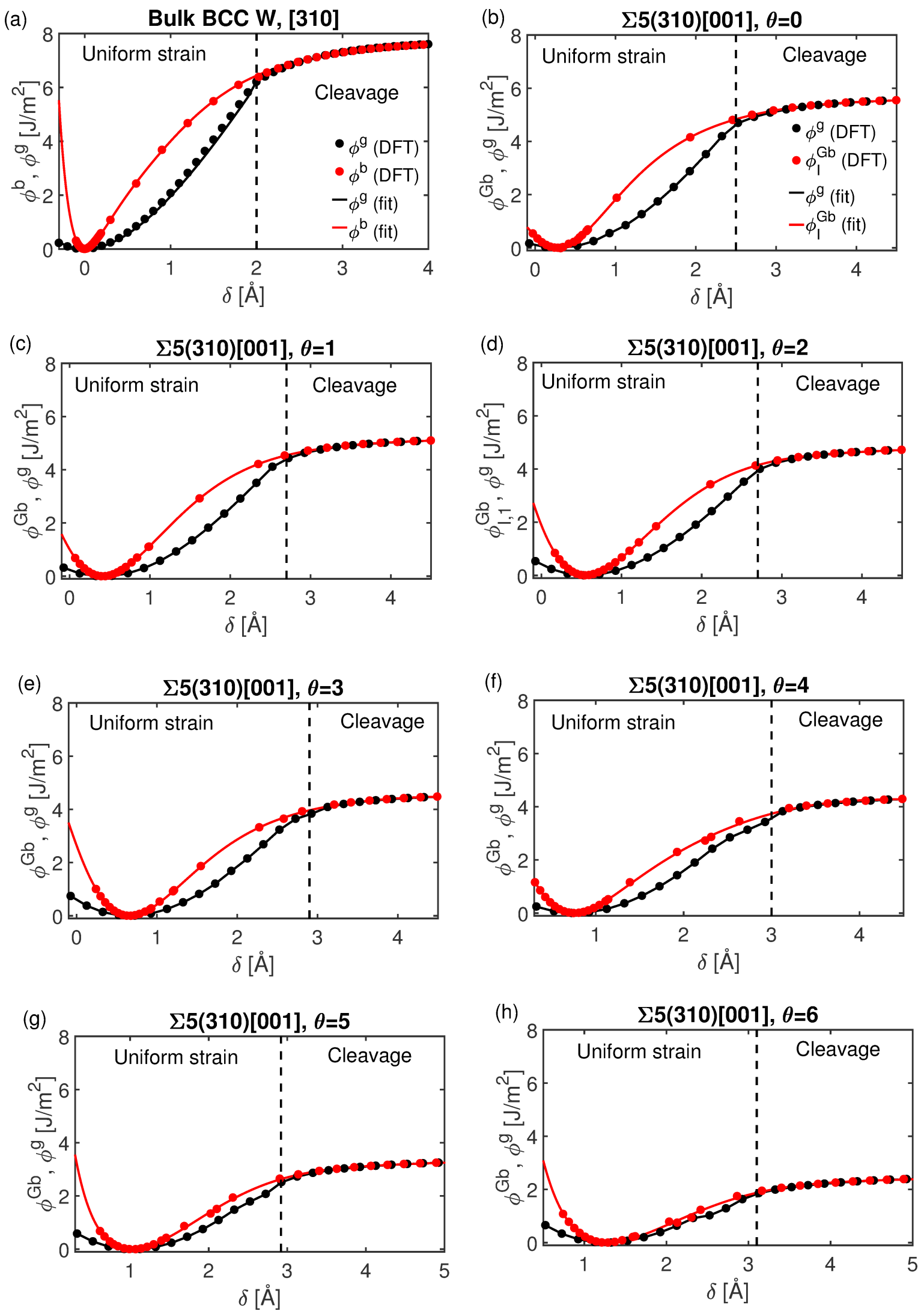

Figure 4: Total supercell energy and excess energy as function of separation for (a) bulk W strained in the [310]-direction, a $\Sigma 5(310)$ [001] grain boundary (b-h) for $\theta=0-6$. The black and red markers correspond to the total supercell and excess energies computed by DFT, whereas the solid lines correspond to the fits of Eqs. (6)-(8) with $\phi_{0}=0$. The vertical dashed line corresponds to the stretch at which cleavage of the supercell initiates. 
Table 1: Fitted xUBER parameters for the excess of perfect bulk, impurity free and inhabited grain boundaries. The parameter $\phi_{0}$ has the unit eV/ $\AA^{2}, C$ has the unit $\mathrm{eV} / \AA^{4}$ and $\delta_{c}$ and $\delta_{0}$ are given in units of $\AA$, whereas $\alpha_{m}$ are dimensionless. It should be noted that $\alpha_{0}=\alpha_{1}=1$ and $\alpha_{2}=0$ for all fits.

\begin{tabular}{|l|cccccccc|}
\hline & $\phi_{0}$ & $C$ & $\alpha_{3}$ & $\alpha_{4}$ & $\alpha_{5}$ & $\alpha_{6}$ & $\delta_{c}$ & $\delta_{0}$ \\
\hline$\phi^{b}$ & 0 & 3.19 & 0.196 & $-1.18 \cdot 10^{-2}$ & $-2.75 \cdot 10^{-3}$ & $7.54 \cdot 10^{-4}$ & 0.389 & 0 \\
$\phi^{G b}(\theta=0)$ & 0.158 & 0.720 & -0.184 & 0.158 & $-3.80 \cdot 10^{-2}$ & $4.23 \cdot 10^{-3}$ & 0.770 & 0.273 \\
$\phi^{G b}(\theta=1)$ & 0.0543 & 0.615 & -0.128 & $1.84 \cdot 10^{-2}$ & $3.24 \cdot 10^{-3}$ & $-9.10 \cdot 10^{-4}$ & 0.708 & 0.412 \\
$\phi^{G b}(\theta=2)$ & -0.0561 & 0.569 & $-8.43 \cdot 10^{-2}$ & $1.81 \cdot 10^{-2}$ & $3.03 \cdot 10^{-3}$ & $2.83 \cdot 10^{-5}$ & 0.762 & 0.554 \\
$\phi^{G b}(\theta=3)$ & -0.158 & 0.591 & -0.112 & $3.65 \cdot 10^{-2}$ & $-5.04 \cdot 10^{-3}$ & $4.56 \cdot 10^{-5}$ & 0.683 & 0.659 \\
$\phi^{G b}(\theta=4)$ & -0.252 & 0.577 & -0.106 & $1.32 \cdot 10^{-2}$ & $6.17 \cdot 10^{-4}$ & $-2.74 \cdot 10^{-3}$ & 0.597 & 0.751 \\
$\phi^{G b}(\theta=5)$ & -0.323 & 0.356 & $-1.87 \cdot 10^{-2}$ & $-8.24 \cdot 10^{-2}$ & $3.06 \cdot 10^{-2}$ & $-3.24 \cdot 10^{-3}$ & 0.744 & 1.02 \\
$\phi^{G b}(\theta=6)$ & -0.404 & 0.277 & $2.01 \cdot 10^{-2}$ & $-3.49 \cdot 10^{-2}$ & $1.86 \cdot 10^{-2}$ & $-9.86 \cdot 10^{-4}$ & 0.825 & 1.28 \\
\hline
\end{tabular}

(a) Traction-separation behaviour

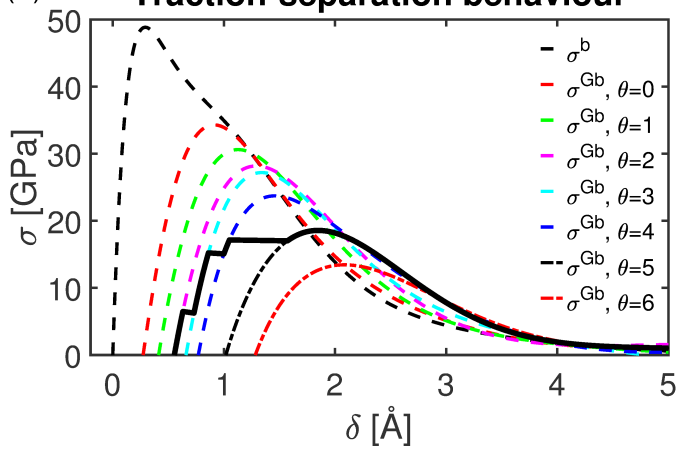

(b) Grand force potential

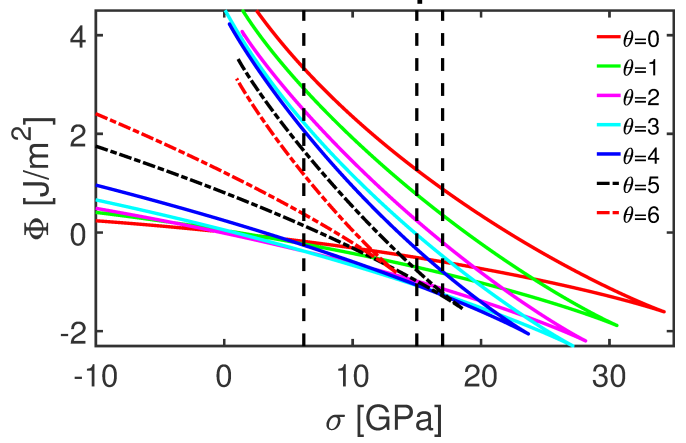

Figure 5: (a) The excess traction-separation behaviour for different phosphorus contents. The black solid curve corresponds to that for infinitely slow tensile rate. (b) Comparison of the grand force potential for the different impurity contents. The locations of cusps correspond to the peak stress positions and the vertical lines correspond to the points at which the transition between different coverage degrees occur.

work of fracture is reduced to $5.1,4.7,4.5$ and $4.1 \mathrm{~J} / \mathrm{m}^{2}$, respectively, and for $\theta=5$ and 6 the work of fracture is further reduced to 3.2 and $2.4 \mathrm{~J} / \mathrm{m}^{2}$, respectively, implying that the increased impurity coverage substantially reduces the work of fracture.

\subsection{Traction-separation behaviour}

Depending on the loading conditions that the material is subjected to, the material response may differ. Mainly, owing to impurity transport, the tensile behaviour may vary substantially if the material is subjected to high or low strain rates, which also influence the peak stress of the material. To investigate these extremes, we consider two cases: (i) a high strain rate for which no impurity diffusion occurs, and (ii) a low strain rate, such that the impurities are allowed diffuse to obtain chemical equilibrium in the cohesive zone. The former of these can be considered to be a special case where the chemical potential for the pure phosphorus is very low and for the latter the strain rate is assumed to be infinitely slow, such that no time scale is considered but rather thermodynamic equilibrium is assumed at all time. For the first case, the traction-separation behaviour for the excess can be obtained simply by the differentiation of (7), which can be seen as the dashed and dash-dotted curves in Figure 5(a). They reveal that the peak stress for a clean grain boundary (i.e. $\theta=0$ ), is lower than that of the perfect bulk in the [310]-direction. Specifically, it reduces from 49 to $34 \mathrm{GPa}$ by the grain boundary introduction, which suggests a weakening of the cohesive strength. This is in line with experimental observations, that there is an affinity towards grain boundary cleavage in tungsten alloys [16]. Thus, because the work of fracture and the peak stress is lower, the threshold for decohesion is reduced, which could imply an embrittlement. However, because it presently is unknown how impurity-free grain boundaries affect the dislocation behaviour, it cannot be concluded whether clean grain boundaries reduce the ductility or not. Nevertheless, this reduction in peak stress is a strong indication that grain boundaries alone may contribute to a weakening, which can promote cleavage. By introducing P-impurities to the grain boundary, the peak stress is gradually reduced from $34 \mathrm{GPa}$ and for $\theta=0$ to $24 \mathrm{GPa}$ for $\theta=4$. Increasing the the $\mathrm{P}$ content further, by inhabiting octahedral sites below the grain boundary interface as indicated in Figure 1(d), reduces the peak stress even further to $13 \mathrm{GPa}$ for $\theta=6$.

To account for the possibility of impurity diffusion it is necessary to vary the impurity content as function of the applied stress. To this end we study how the grand force potential (Eq. (1)) varies with stress, with the chemical potential obtained from Eq. (9). This approach gives an indication of how the traction-separation properties are affected by the varying impurity coverage during the gradual crack opening. In Figure 5(b) it is seen that from zero stress up to about $6.2 \mathrm{GPa}$, the lowest grand force potential configuration is that of $\theta=2$, which concurs with the fact impurities preferentially segregate at the grain boundary. If the stress is increased above 6.2 GPa the curves for $\theta=2$ and $\theta=3$ cross such that the cohesive zone separation increases discontinuously to accommodate diffusing impurities to the cohesive zone, which is represented by the first horizon- 
tal plateau observed in Figure 5(a). As shown by the vertical lines in Figure 5(b) this type of transition is repeated again at 15 and $17 \mathrm{GPa}$, such that the grain boundary coverage increases to $\theta=4$ and finally $\theta=5$, respectively. Thus, as indicated in Figure 5(a), the peak stress corresponds to that of $\theta=5(18.5 \mathrm{GPa})$, which suggests a significant reduction in cohesive strength in the presence of solute and diffusing P-impurities in the grain boundaries. Compared to the perfect bulk tungsten this corresponds to a reduction to less than half in peak stress, which is a strong indication that the material becomes increasingly susceptible to grain boundary cleavage. Because no transformation to the system corresponding to $\theta=6$ is observed before the maximum stress of the $\theta=5$ curve is reached, it does not affect the peak stress. However, because the grand force potential for $\theta=6$ is lower than that for $\theta=5$ after the peak stress has been reached, it is expected that $\mathrm{P}$ atoms will completely cover both free surfaces after the cleavage has occurred.

Phosphorus diffusion in single-crystalline tungsten is characterised by a high activation energy $(Q=510 \mathrm{~kJ} / \mathrm{mol}$ for $T>2150 \mathrm{~K})$ and a diffusivity $D_{0} \sim 27 \mathrm{~cm}^{2} / \mathrm{s}$ [85], which are comparable to the tungsten vacancy based self-diffusion ( $\left.Q=600 \mathrm{~kJ} / \mathrm{mol}, D_{0}=19 \mathrm{~cm}^{2} / \mathrm{s}[86]\right)$. We expect the diffusion to be relatively high following the increased vacancy clustering due to irradiation and the high operating temperature in fusion reactors. Moreover, transport along surfaces of nano and micro cracks will occur rapidly. Because the peak stress is gradually lowered as the impurities agglomerate at the stressed zone, even a static load can give rise to crack propagation. This may result in delayed cracking of the material even if the stress levels initially are below the peak stress. Although the modelling does not predict any time scale related to the loading rate that can be used to predict which approach is more appropriate to use, we expect the situation to end up somewhere between the results of low chemical potential and infinitely slow strain rate modelling. Thus, a peak stress in the range $18-34 \mathrm{GPa}$ is expected.

\section{Summary and conclusions}

In the present work we have studied the influence of phosphorus impurities on the grain boundary cohesion of a $\Sigma 5(310)[001]$ grain boundary in tungsten by means of DFT modelling in conjunction with a thermodynamic analysis of cleavage performed at constant chemical potential. Pure (i.e. impurity free) grain boundaries have a lower cohesive strength than the perfect bulk and the work of fracture and peak stress decrease as the impurity content gradually increases. Thus, phosphorus segregated in the grain boundary contributes to the promotion of cleavage. These results concur with experimental observations.

To understand the mechanism behind the impurity induced cohesive reduction of the grain boundary, we perform bonding electron density and Bader charge analyses. The analyses reveal that there is an electron transfer from the surrounding tungsten to the impurity atoms. Based on the electron density distribution it is concluded that no significant covalent bonding is formed between the matrix and the impurity atoms. Thus, the bonding is of polar character and relatively weak, such that the impurities act as negatively charged screened ions weakening the grain boundaries. These findings are in line with predictions based on the unified theory of Cottrell [84].

The modelling shows that the impurities preferentially segregate as in the grain boundaries as opposed to in the bulk, which concurs with experimental observations. The thermodynamic analysis predicts that further impurities will diffuse to the loaded region in order to retain thermodynamic equilibrium at constant chemical potential as the stress increases. This leads to a gradual reduction in the grain boundary peak stress. Thus, depending on the loading conditions (e.g. loading rate, crack propagation speed etc.) the $\mathrm{P}$ atoms may agglomerate at the crack tip and gradually reduce the peak stress of the cohesive zone. For slow or static loading this may result in delayed cracking that is diffusion driven.

\section{Acknowledgements}

The authors gratefully acknowledge funding from the Swedish Research Council (grant no. 2016-04162) and from the Crafoord Foundation (grant no. 2016-0740). The simulations in this work were performed using computational resources provided by the Swedish National Infrastructure for Computing (SNIC) at the National Supercomputer Centre (NSC), Linköping University and at the High Performance Computing Center North (HPC2N), Umeå University.

[1] E. Lassner, W. D. Schubert, Tungsten: Properties, Chemistry, Technology of the Element, Alloys, and Chemical Compounds, Springer US, 2012.

[2] M. Rieth, S. L. Dudarev, S. M. G. de Vicente, J. Aktaa, T. Ahlgren, S. Antusch, D. Armstrong, M. Balden, N. Baluc, M.-F. Barthe, W. Basuki, M. Battabyal, C. Becquart, D. Blagoeva, H. Boldyryeva, J. Brinkmann, M. Celino, L. Ciupinski, J. Correia, A. D. Backer, C. Domain, E. Gaganidze, C. Garcia-Rosales, J. Gibson, M. Gilbert, S. Giusepponi, B. Gludovatz, H. Greuner, K. Heinola, T. Höschen, A. Hoffmann, N. Holstein, F. Koch, W. Krauss, H. Li, S. Lindig, J. Linke, C. Linsmeier, P. Lopez-Ruiz, H. Maier, J. Matejicek, T. Mishra, M. Muhammed, A. Munoz, M. Muzyk, K. Nordlund, D. Nguyen-Manh, J. Opschoor, N. Ordas, T. Palacios, G. Pintsuk, R. Pippan, J. Reiser, J. Riesch, S. G. Roberts, L. Romaner, M. Rosinski, M. Sanchez, W. Schulmeyer, H. Traxler, A. Urena, J. van der Laan, L. Veleva, S. Wahlberg, M. Walter, T. Weber, T. Weitkamp, S. Wurster, M. A. Yar, J. H. You, A. Zivelonghi, Recent progress in research on tungsten materials for nuclear fusion applications in europe, J. Nucl. Mater. 432 (2013) 482 - 500.

[3] R. A. Causey, T. J. Venhaus, The use of tungsten in fusion reactors: a review of the hydrogen retention and migration properties, Phys. Scripta 2001 (T94) 9.

[4] L. Cao, Z. Zhou, D. Yao, EAST full tungsten divertor design, J. Fusion Energy 34 (6) (2015) 1451-1456.

[5] S. Wurster, N. Baluc, M. Battabyal, T. Crosby, J. Du, C. Garca-Rosales, A. Hasegawa, A. Hoffmann, A. Kimura, H. Kurishita, R. Kurtz, H. Li, S. Noh, J. Reiser, J. Riesch, M. Rieth, W. Setyawan, M. Walter, J.-H. You, R. Pippan, Recent progress in R\&D on tungsten alloys for divertor structural and plasma facing materials, J. Nucl. Mater. 442 (2013) S181 S189.

[6] V. Philipps, Tungsten as material for plasma-facing components in fusion devices, J. Nucl. Mater. 415 (2011) S2 - S9.

[7] P. Beardmore, D. Hull, Deformation and fracture of tungsten single crystals, J. Less-Common Met. 9 (3) (1965) 168.

[8] A. Joshi, D. F. Stein, Intergranular brittleness studies in tungsten using auger spectroscopy, Metall. Trans. 1 (9) (1970) 2543.

[9] S. Pestchanyi, J. Linke, Simulation of cracks in tungsten under ITER specific transient heat loads, Fus. Engng. Design 82 (2007) 1657 - 1663.

[10] S. Pestchanyi, I. Garkusha, I. Landman, Simulation of residual thermostress in tungsten after repetitive ELM-like heat loads, Fus. Engng. Design 86 (2011) 1681. 
[11] D. Rupp, S. M. Weygand, Loading rate dependence of the fracture toughness of polycrystalline tungsten, J. Nucl. Mater. 417 (2011) 477 - 480.

[12] A. Giannattasio, Z. Yao, E. Tarleton, S. Roberts, Brittle-ductile transitions in polycrystalline tungsten, Philos. Mag. 90 (30) (2010) 3947-3959.

[13] T. Tanno, A. Hasegawa, J.-C. He, M. Fujiwara, S. Nogami, M. Satou, T. Shishido, K. Abe, Effects of transmutation elements on neutron irradiation hardening of tungsten, Mater. Trans. 48 (9) (2007) 2399-2402.

[14] P. Gumbsch, Brittle fracture and the brittle-to-ductile transition of tungsten, J. Nucl. Mater. 323 (2003) 304 - 312

[15] T. H. Loi, J. P. Morniroli, M. Gantois, Brittle fracture of polycrystalline tungsten, J. Mater. Sci. 20 (1985) 199-206.

[16] B. Gludovatz, S. Wurster, T. Weingärtner, A. Hoffmann, R. Pippan, Influence of impurities on the fracture behaviour of tungsten, Philos. Mag. 91 (22) (2011) 3006-3020.

[17] P. A. T. Olsson, K. Kese, M. Kroon, A.-M. Alvarez Holston, Ab initio -based fracture toughness estimates and transgranular traction-separation modelling of zirconium hydrides, Modell. Simul. Mater. Sci. Eng. 23 (4) (2015) 045015 .

[18] P. A. T. Olsson, K. Kese, A.-M. Alvarez Holston, On the role of hydrogen filled vacancies on the embrittlement of zirconium: An ab initio investigation, J. Nucl. Mater. 467 (2015) $311-319$.

[19] P. A. T. Olsson, M. Mrovec, M. Kroon, First principles characterisation of brittle transgranular fracture of titanium hydrides, Acta Mater. 118 (2016) $362-373$.

[20] M. A. Gibson, C. A. Schuh, A survey of ab-initio calculations shows that segregation-induced grain boundary embrittlement is predicted by bondbreaking arguments, Scr. Mater. 113 (2016) $55-58$.

21] M. A. Gibson, C. A. Schuh, A compilation of ab-initio calculations of embrittling potencies in binary metallic alloys, Data in Brief 6 (2016) $143-148$.

[22] A. Tahir, R. Janisch, A. Hartmaier, Hydrogen embrittlement of a carbon segregated symmetrical tilt grain boundary in $\alpha$-Fe, Mater. Sci. Engng. A 612 (2014) $462-467$

[23] L. Zhong, R. Wu, A. J. Freeman, G. B. Olson, Effects of Mn additions on the P embrittlement of the Fe grain boundary, Phys. Rev. B 55 (1997) $11133-11137$.

[24] W. T. Geng, A. J. Freeman, R. Wu, G. B. Olson, Effect of Mo and Pd on the grain-boundary cohesion of Fe, Phys. Rev. B 62 (2000) 6208-6214.

[25] K.-D. Bauer, M. Todorova, K. Hingerl, J. Neugebauer, A first principles investigation of zinc induced embrittlement at grain boundaries in bcc iron, Acta Mater. 90 (2015) 69 - 76

[26] M. Yamaguchi, First-principles study on the grain boundary embrittlement of metals by solute segregation: Part I. iron (Fe)-solute (B, C, P, and S) systems, Metall. Mater. Trans. A 42 (2) (2011) 319-329.

[27] R. Wu, A. J. Freeman, G. B. Olson, First principles determination of the effects of phosphorus and boron on iron grain boundary cohesion, Science 265 (5170) (1994) 376

[28] J. Wang, R. Janisch, G. K. Madsen, R. Drautz, First-principles study of carbon segregation in bcc iron symmetrical tilt grain boundaries, Acta Mater. 115 (2016) $259-268$.

[29] A. M. Tahir, R. Janisch, A. Hartmaier, Ab initio calculation of traction separation laws for a grain boundary in molybdenum with segregated $\mathrm{C}$ impurites, Modell. Simul. Mater. Sci. Eng. 21 (2013) 075005.

[30] R. Janisch, C. Elsässer, Segregated light elements at grain boundaries in niobium and molybdenum, Phys. Rev. B 67 (2003) 224101

[31] R. Janisch, C. Elsässer, Interstitial impurities at grain boundaries in metals: insight from atomistic calculations, Int. J. Mater. Res. 100 (2009) 1488-1493.

[32] G. L. Krasko, Effect of impurities on the electronic structure of grain boundaries and intergranular cohesion in tungsten, Int. J. Refract. Met. H. 12 (5) (1993) $251-260$

[33] M. Grujicic, H. Zhao, G. Krasko, Atomistic simulation of $\Sigma 3(111)$ grain boundary fracture in tungsten containing various impurities, Int. J. Refract. Met. H. 15 (5) (1997) $341-355$.

[34] J. R. Rice, J.-S. Wang, Embrittlement of interfaces by solute segregation, Mater. Sci. Engng. A 107 (1989) 23 - 40.

[35] H. B. Zhou, S. Jin, Y. Zhang, G. H. Lu, Effects of hydrogen on a tungsten grain boundary: A first-principles computational tensile test, Prog. Nat. Sci.: Mater. Int. 21 (3) (2011) 240 - 245

[36] W. Setyawan, R. J. Kurtz, Ab initio study of H, He, Li and Be impurity effect in tungsten $\Sigma 3\{112\}$ and $\Sigma 27\{552\}$ grain boundaries, J. Phys.: Cond.
Matter 26 (13) (2014) 135004

[37] L. Zhi-Wu, K. Xiang-Shan, Liu-Wei, L. Chang-Song, F. Qian-Feng, Segregation of alloying atoms at a tilt symmetric grain boundary in tungsten and their strengthening and embrittling effects, Chin. Phys. B 23 (10) (2014) 106107.

[38] W. Setyawan, R. J. Kurtz, Effects of transition metals on the grain boundary cohesion in tungsten, Scr. Mater. 66 (8) (2012) $558-561$.

[39] D. Scheiber, R. Pippan, P. Puschnig, A. Ruban, L. Romaner, Ab-initio search for cohesion-enhancing solute elements at grain boundaries in molybdenum and tungsten, Int. J. Refract. Met. H. 60 (2016) 75 - 81.

[40] D. Scheiber, R. Pippan, P. Puschnig, L. Romaner, Ab initio calculations of grain boundaries in bcc metals, Model. Simul. Mater. Sci. Engng. 24 (3) (2016) 035013

[41] Z. Pan, L. J. Kecskes, Q. Wei, The nature behind the preferentially embrittling effect of impurities on the ductility of tungsten, Comput. Mater. Sci. 93 (2014) $104-111$.

[42] D. Dugdale, Yielding of steel sheets containing slits, J. Mech. Phys. Solids 8 (2) (1960) $100-104$.

[43] G. Barenblatt, The mathematical theory of equilibrium cracks in brittle fracture, Adv. Appl. Mech. 7 (1962) 55-129.

[44] A. Van der Ven, G. Ceder, The thermodynamics of decohesion, Acta Mater. 52 (5) (2004) 1223 - 1235.

[45] R. Dingreville, J. Qu, M. Cherkaoui, Surface free energy and its effect on the elastic behavior of nano-sized particles, wires and films, J. Mech. Phys. Solids 53 (8) (2005) 1827 - 1854.

[46] R. A. Enrique, A. Van der Ven, Solute embrittlement of SiC, J. Appl. Phys. 116 (11) (2014) 113504

[47] R. A. Enrique, A. Van der Ven, Decohesion models informed by firstprinciples calculations: The ab initio tensile test, J. Mech. Phys. Solids 107 (2017) 494 - 508

[48] R. A. Enrique, A. Van der Ven, Traction curves for the decohesion of covalent crystals, Appl. Phys. Lett. 110 (2) (2017) 021910.

[49] J. M. Sanchez, F. Ducastelle, D. Gratias, Generalized cluster description of multicomponent systems, Physica A 128 (12) (1984) 334 - 350.

[50] J. M. Sanchez, Cluster expansion and the configurational theory of alloys, Phys. Rev. B 81 (2010) 224202.

[51] Q. Xu, A. Van der Ven, First-principles investigation of metal-hydride phase stability: The Ti-H system, Phys. Rev. B 76 (2007) 064207.

[52] S. Baroni, P. Giannozzi, E. Isaev, Density-functional perturbation theory for quasi-harmonic calculations, Reviews in Mineralogy and Geochemistry 71 (1) (2010) 39-57.

[53] G. Kresse, J. Hafner, $A b$ initio molecular dynamics for liquid metals, Phys. Rev. B 47 (1993) 558-561.

[54] G. Kresse, J. Hafner, Ab initio molecular-dynamics simulation of the liquid-metal amorphous-semiconductor transition in germanium, Phys. Rev. B 49 (1994) 14251-14269.

[55] G. Kresse, J. Furthmüller, Efficient iterative schemes for ab initio totalenergy calculations using a plane-wave basis set, Phys. Rev. B 54 (1996) 11169-11186.

[56] G. Kresse, J. Furthmüller, Efficiency of ab-initio total energy calculations for metals and semiconductors using a plane-wave basis set, Comput. Mater. Sci. 6 (1) (1996) 15 - 50.

[57] P. E. Blöchl, Projector augmented-wave method, Phys. Rev. B 50 (1994) 17953-17979.

[58] G. Kresse, D. Joubert, From ultrasoft pseudopotentials to the projector augmented-wave method, Phys. Rev. B 59 (1999) 1758-1775.

[59] M. Dion, H. Rydberg, E. Schröder, D. C. Langreth, B. I. Lundqvist, Van der Waals density functional for general geometries, Phys. Rev. Lett. 92 (2004) 246401.

[60] M. Dion, H. Rydberg, E. Schröder, D. C. Langreth, B. I. Lundqvist, Erratum: Van der Waals density functional for general geometries [phys. rev. lett. 92 , 246401 (2004)], Phys. Rev. Lett. 95 (2005) 109902.

[61] J. Klimes, D. R. Bowler, A. Michaelides, Chemical accuracy for the van der Waals density functional, J. Phys.: Cond. Matter 22 (2) (2010) 022201

[62] J. Klimes, D. R. Bowler, A. Michaelides, Van der Waals density functionals applied to solids, Phys. Rev. B 83 (2011) 195131

[63] J. Carrasco, W. Liu, A. Michaelides, A. Tkatchenko, Insight into the description of van der waals forces for benzene adsorption on transition metal (111) surfaces, J. Chem. Phys 140 (8) (2014) 084704

[64] F. Bachhuber, J. von Appen, R. Dronskowski, P. Schmidt, T. Nilges, 
A. Pfitzner, R. Weihrich, Van der waals interactions in selected allotropes of phosphorus, Z. Kristallog. Cryst. Mater. 230 (2015) 107 - 115.

[65] S. Appalakondaiah, G. Vaitheeswaran, S. Lebègue, N. E. Christensen, A. Svane, Effect of van der Waals interactions on the structural and elastic properties of black phosphorus, Phys. Rev. B 86 (2012) 035105.

[66] H. J. Monkhorst, J. D. Pack, Special points for brillouin-zone integrations, Phys. Rev. B 13 (1976) 5188-5192.

[67] M. Methfessel, A. T. Paxton, High-precision sampling for brillouin-zone integration in metals, Phys. Rev. B 40 (1989) 3616-3621.

[68] B. N. Dutta, B. Dayal, Lattice constants and thermal expansion of palladium and tungsten up to $878^{\circ} \mathrm{C}$ by x-ray method, Phys. Status Solidi B 3 (12) (1963) 2253-2259.

[69] L. Cartz, S. R. Srinivasa, R. J. Riedner, J. D. Jorgensen, T. G. Worlton, Effect of pressure on bonding in black phosphorus, J. Chem. Phys. 71 (4) (1979) 1718-1721.

[70] B. M. Cossairt, C. C. Cummins, A. R. Head, D. L. Lichtenberger, R. J. F. Berger, S. A. Hayes, N. W. Mitzel, G. Wu, On the molecular and electronic structures of $\mathrm{AsP}_{3}$ and $\mathrm{P}_{4}$, J. Am. Chem. Soc. 132 (24) (2010) 8459-8465.

[71] G. H. Campbell, J. Belak, J. A. Moriarty, Atomic structure of the $\Sigma 5$ (310)/[001] symmetric tilt grain boundary in molybdenum, Acta Mater. 47 (1999) 3977 - 3985.

[72] G. H. Campbell, S. M. Foiles, P. Gumbsch, M. Rühle, W. E. King, Atomic structure of the (310) twin in niobium: Experimental determination and comparison with theoretical predictions, Phys. Rev. Lett. 70 (1993) 449 452.

[73] G. H. Campbell, J. Belak, J. A. Moriarty, Atomic structure of the $\Sigma 5$ $(310) /[001]$ symmetric tilt grain boundary in tantalum, Scr. Mater. 43 (7) (2000) $659-664$.

[74] T. Ochs, O. Beck, C. Elsässer, B. Meyer, Symmetrical tilt grain boundaries in body-centred cubic transition metals: An ab initio local-densityfunctional study, Philos. Mag. A 80 (2) (2000) 351-372.

[75] O. Nguyen, M. Ortiz, Coarse-graining and renormalization of atomistic binding relations and universal macroscopic cohesive behavior, J. Mech. Phys. Solids 50 (8) (2002) 1727 - 1741.

[76] J. H. Rose, J. Ferrante, J. R. Smith, Universal binding energy curves for metals and bimetallic interfaces, Phys. Rev. Lett. 47 (1981) 675-678.

[77] J. H. Rose, J. R. Smith, J. Ferrante, Universal features of bonding in metals, Phys. Rev. B 28 (1983) 1835-1845.

[78] J. A. Nelder, R. Mead, A simplex method for function minimization, Comput. J. 7 (4) (1965) 308-313.

[79] J. P. Perdew, K. Burke, M. Ernzerhof, Generalized gradient approximation made simple, Phys. Rev. Lett. 77 (1996) 3865-3868.

[80] J. P. Perdew, K. Burke, M. Ernzerhof, Generalized gradient approximation made simple [erratum], Phys. Rev. Lett. 78 (1997) 1396-1396.

[81] S. Zumdahl, D. J. DeCoste, Chemical Principles, Cengage Learning, 2013.

[82] R. Bader, Atoms in Molecules: A Quantum Theory, International Ser. of Monogr. on Chem, Clarendon Press, 1994

[83] W. Tang, E. Sanville, G. Henkelman, A grid-based bader analysis algorithm without lattice bias, J. Phys.: Cond. Matter 21 (8) (2009) 084204.

[84] A. H. Cottrell, Unified theory of effects of segregated interstitials on grain boundary cohesion, Mater. Sci. Tech. 6 (9) (1990) 806-810.

[85] H. Jehn, J. Jouanne, E. Koch, G. Bär, E. Best, E. Koch, W Tungsten: Supplement Volume A 5 b Metal, Chemical Reactions with Nonmetals Nitrogen to Arsenic, Gmelin Handbook of Inorganic and Organometallic Chemistry - 8th edition, Springer Berlin Heidelberg, 2013.

[86] D. R. Askeland, The Science and Engineering of Materials, SI Edition, 3rd Edition, Stanley-Thornes, 1998. 